\title{
Descripción de la jerarquía de controles frente al peligro químico por exposición a compuestos orgánicos volátiles generados por procesos de pintura en el sector industrial ${ }^{1}$
}

\author{
Carlos Giovanny Rincón Cuervo²
}

Adriana María Ortiz Vásquez ${ }^{3}$

doi:10.11144/Javeriana.IE17-1.djcp

Como citar: Rincón Cuervo CG, Ortiz Vásquez AM. Descripción de la jerarquía de controles frente al peligro químico por exposición a compuestos orgánicos volátiles generados por procesos de pintura en el sector industrial. Investig Enferm. Imagen Desarr. 2015;17(1):149-69. http://dx.doi.org/10.11144/Javeriana.IE17-1.djcp

1. Artículo original de investigación, derivado del trabajo de grado con mención de honor del programa de Especialización de Salud Ocupacional de la Pontificia Universidad Javeriana. Recibido: 24 de julio de 2014. Aprobado: 30 de septiembre de 2014.

2. Ingeniero químico, Universidad Industrial de Santander, Bucaramanga, Colombia. Especialista en Salud Ocupacional, Pontificia Universidad Javeriana, Bogotá, Colombia. Contratista de la Dirección de Desarrollo Tecnológico e Innovación de Colciencias. Correo electrónico: carlosgianny@hotmail.com

3. Ingeniera industrial, Universidad de América, Bogotá, Colombia. Especialista en Salud Ocupacional, Pontificia Universidad Javeriana, Bogotá, Colombia. Ingeniera de Seguridad, Salud y Medio Ambiente en SNC-Lavalin. Correo electrónico: adrianamariaov@hotmail.com 


\section{Resumen}

En los procesos de pintura de las industrias automotriz, metalmecánica, transformación de madera y construcción se utilizan varias sustancias químicas generadoras de compuestos orgánicos volátiles (COV), las cuales producen efectos adversos en la salud humana y están relacionadas con el grado de toxicidad del agente químico y del tiempo de exposición. El objetivo de este trabajo fue describir la jerarquía de controles existentes frente al peligro químico, por exposición a COV en las industrias mencionadas, con el fin de orientar a las partes interesadas en la implementación de medidas en el ambiente de trabajo y en el individuo, que lleven a un manejo seguro y que permita mitigar los efectos adversos derivados del uso de los COV. La metodología se fundamentó en recopilar y analizar la información existente en higiene industrial y salud en el trabajo de organizaciones como American Conference of Industrial Hygienists, National Institute for Occupational Safety and Health, Occupational Safety and Health Administration, International Agency for Research on Cancer, American National Standards Institute, entre otras. Como resultado, se identificaron 107 sustancias químicas como las principales componentes de los productos usados en los procesos de pintura, de los cuales 59 son COV, 12 están en los incluidos dentro de los grupos 1 y 2 de la International Agency for Research on Cancer, 24 cuentan con valor limite permisible por debajo de 100 partes por millón y 19 se encuentran sobre dicho valor; por ello se requieren controles de sustitución, ingeniería e intervenciones de tipo administrativo.

Palabras clave: control de riesgo; compuestos orgánicos volátiles (COV); pintura; exposición a compuestos químicos

\section{Description of the Hierarchy of Controls Regarding Chemical Hazard for Exposure to Volatile Organic Compounds Generated by Painting Processes in Industry}

\section{Abstract}

In the painting process of the industries such as automotive, metalworking, woodworking and construction different kind of chemicals are used. Some of them produce volatile organic compounds (VOC) and cause adverse effects on human health, which are related to the degree of toxicity of the chemical and the time of exposure. The target was describe the hierarchy of controls that were performed against chemical hazards related to exposure to volatile organic compounds, in order to advice stakeholders in the implementation of measures at individual and work environment that lead to the safe handling and counteract the adverse effects from the use of VOCs. The methodology was based on collecting and analyzing information from Industrial Hygiene and Health at work of organizations such as American Conference of Industrial Hygienists, National Institute for Occupational Safety and Health, Occupational Safety and Health Administration, International Agency for Research on Cancer, American National Standards Institute, among others. The result of this research identified 107 chemicals as the main components of the products used in the painting process. Among them 59 are VOC, 12 are in groups 1 and 2 of International Agency for Research on Cancer, 24 have TLV below 100 ppm and 19 are on that value, therefore, controls of substitution, engineering and administrative interventions are required.

Keywords: risk management; volatile organic compounds (VOC's); paint; chemical compound exposure 


\section{Descrição da jerarquia de controles pela exposição ao perigo quimico por compostos orgânicos voláteis gerados de processos de pintura no setor industrial}

\section{Resumo}

No processo de pintura automotiva, metalurgia, marcenaria e construção, várias substâncias químicas que geram compostos orgânicos voláteis (COV) são usados que causam efeitos adversos na saúde humana, que estão relacionadas com o grau de toxicidade do produto químico e o tempo de exposição. O objetivo era fazer com que a descrição da hierarquia de controles em frente ao perigo químico, exposição a compostos orgânicos voláteis nas indústrias mencionadas acima, a fim de orientar as partes interessadas na implementação de medidas no ambiente de trabalho e do indivíduo, que levam a uma condução segura e ajudar a mitigar os efeitos adversos decorrentes da utilização de compostos orgânicos voláteis. A metodologia foi baseada na coleta e análise de informações sobre higiene industrial e saúde no trabalho de organizações como a Conferência Americana de Higienistas Industriais, Instituto Nacional de Segurança e Saúde Ocupacional, Occupational Safety and Health Administration, Agência Internacional para Pesquisa em Câncer, American National Standards Institute, entre outros. Como resultado, 107 substâncias químicas como os principais componentes dos produtos utilizados no processo de pintura, dos quais 59 são compostos orgânicos voláteis, 12 estão em incluídos nos grupos 1 e 2 Agência Internacional para Pesquisa em Câncer, 24 têm TLV abaixo identificado 100 ppm e 19 de mentir sobre esse valor, segundo o qual as intervenções controles de nível de substituição, engenharia e administrativas necessárias.

Palavras chave: controle de risco; compostos orgânicos voláteis (COV); pintura; exposição a produtos químicos 


\section{Introducción}

En los últimos años se ha incrementado el interés de las organizaciones por promover ambientes laborales seguros y saludables (1) que permitan beneficiar tanto a las empresas como a sus colaboradores, mediante la gestión integral de los peligros, la minimización de los riesgos y la promoción de políticas de seguridad en el trabajo (2), cuyo objetivo es prevenir los accidentes y enfermedades laborales.

Entidades como la Organización Internacional del Trabajo promueven los derechos de los trabajadores, fomentan oportunidades de trabajo decente, mejoran las directrices internacionales frente a la protección social y fortalecen el diálogo, al abordar los temas relacionados con el trabajo formulando politicas y programas internacionales que aportan a la mejora de las condiciones de trabajo y de vida, al tiempo que realizan actividades de formación, educación e investigación.

Dentro de los peligros a los cuales se encuentran expuestos los trabajadores, uno de los más comunes está dado por el manejo (3) y uso de sustancias químicas $(4,5)$, utilizadas en la mayoría de los procesos industriales y cuyos efectos en la salud humana dependen, entre otras, de la toxicidad del agente químico (6), el tiempo de exposición y el grado de implementación de la jerarquía de controles $(7,8)$.

Fueron de particular interés en el estudio los compuestos orgánicos volátiles (COV), dado su amplio uso, sus características particulares y sus conocidos efectos en la salud $(9,10)$. Como tema central se presentó una descripción de la jerarquía de los controles (11), en cuanto a sustitución, controles de ingeniería y controles administrativos, con el ánimo de establecer un documento de consulta para que las partes interesadas puedan adoptar, en algún momento determinado, medidas encaminadas a reducir o mitigar los efectos de la exposición laboral durante el manejo de estas sustancias en los proceso de pintura propios de las industrias automotriz, metalmecánica, transformación de madera y construcción $(12,13)$.

\section{Objetivo general}

Describir la jerarquía de controles existentes frente al peligro químico, por exposición a COV generados por procesos de pintura en el sector industrial.

\section{Objetivos especificos}

- Identificar en los procesos industriales de pintura las principales sustancias quimicas, que generan exposición a COV.

- Presentar los modelos y alternativas aplicables en los procesos industriales de pintura que lleven a la sustitución de sustancias químicas generadoras de COV.

- Describir los controles de ingeniería propuestos por la ventilación industrial enfocados en los procesos de aplicación de pintura en el ámbito industrial.

- Indicar los controles administrativos referentes a la prevención de enfermedades laborales por exposición a COV en los procesos de pintura. 


\section{Material y método}

La metodología utilizada para la realización del estudio fue integrativa fundamentada en la recopilación y análisis de bibliografía nacional y mundial referente a procesos industriales de pintura en las industrias objeto de estudio en los últimos diez años, higiene industrial, hojas de seguridad, química orgánica, ventilación industrial, entre otros; así mismo, se realizaron consultas de publicaciones de organizaciones internacionales relacionadas con la salud en el trabajo como American Conference of Industrial Hygienists (ACGIH), National Institute for Occupational Safety and Health (NIOSH), Occupational Safety and Health Administration (OSHA), International Agency for Research on Cancer (IARC), Instituto Nacional de Seguridad e Higiene en el Trabajo (INSHT), Organización Internacional del Trabajo, Organización Mundial de la Salud, National Fire Protection Association (NFPA), Institut National de la Recherche Scientifique et de Sécurité pour la Prévention des Accidents de Travail et des Maladies Professionnelles (INRS), Environmental Protection Agency (EPA) e Institut für Arbeitsschuzt der Deutschen Gesetzlichen Unfallversicherung (IFA). Igualmente, guías sectoriales, sistema de vigilancia de salud pública, sistema de vigilancia de cáncer ocupacional, legislación nacional sobre manejo de sustancias químicas, guías de atención basadas en la evidencia y normas técnicas nacionales e internacionales.

La revisión de las fuentes de consulta permitió, entre otras cosas, identificar las principales sustancias químicas utilizadas en la formulación de productos usados en las actividades de pintura de las industrias de interés ofrecidos por varios fabricantes, la clasificación de estas como carcinógenas según el IARC y la inclusión en el Sistema de Vigilancia del Cáncer Ocupacional, a fin de implementar estrategias de intervención en los trabajadores expuestos a los COV identificados en este estudio.

Esta jerarquía se basó en la propuesta por la norma ANSI Z10 (11), la cual proporciona información sobre la implementación de los sistemas de gestión de seguridad y salud ocupacional en las organizaciones.

\section{Resultados y discusión}

Mediante la revisión de bibliografía referente a las actividades relacionadas con los procesos de pintura (14) de las industrias automotriz, metalmecánica, transformación de la madera y construcción, los productos químicos utilizados y sus hojas de seguridad, se identificaron un total de 107 sustancias químicas cuyo uso es altamente significativo.

Del total de las sustancias identificadas, el 55\% son COV, es decir, más de la mitad de los productos usados los contienen. Este valor es importante, dados los efectos agudos y crónicos que estas sustancias generan en la salud de los trabajadores (15). Se evidenció que un 16\% de las sustancias son reconocidas como carcinógenas según IARC (16) y un 5\% están identificadas en el Sistema de Vigilancia del Cáncer Ocupacional (figura 1).

En el sector automotor (17) se identificaron 42 sustancias, 29 de ellas COV, 10 son carcinógenas según IARC y 4 están incluidas dentro del Sistema de Vigilancia Epidemiológica de Cáncer Ocupacional (figura 2). 
En la industria metalmecánica $(18,19)$ se identificaron en total 43 sustancias, de las cuales 22 son generadoras de COV, 6 están identificadas como carcinógenas en IARC y 2 están registradas en el Sistema de Vigilancia Epidemiológica del Cáncer Ocupacional (20) (figura 3).

En la industria de la transformación de madera (21-24) se identificaron 52 sustancias, de las cuales 36 generan COV, 8 están identificadas

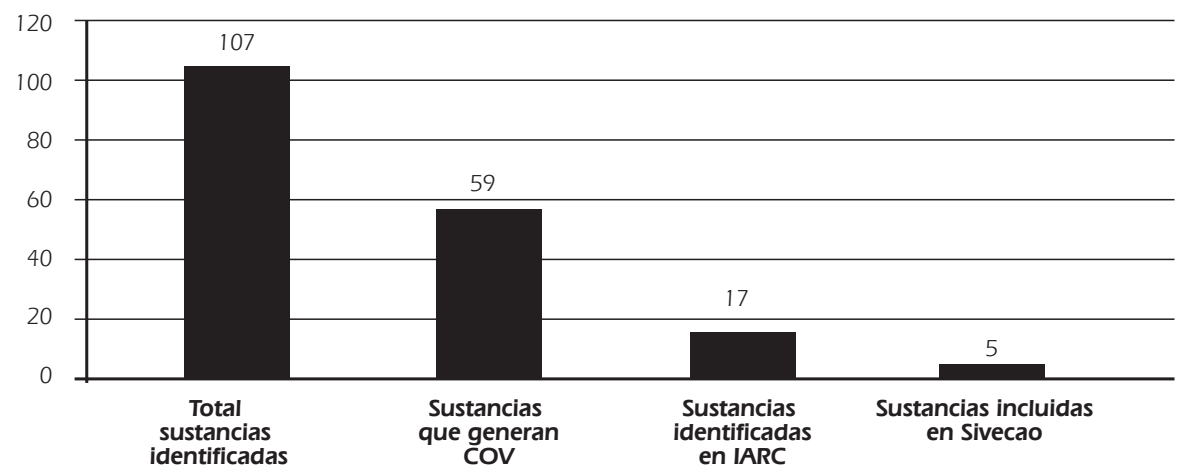

Figura 1. Sustancias identificadas utilizadas en los procesos de pintura de las industrias de interés

Fuente: elaboración propia

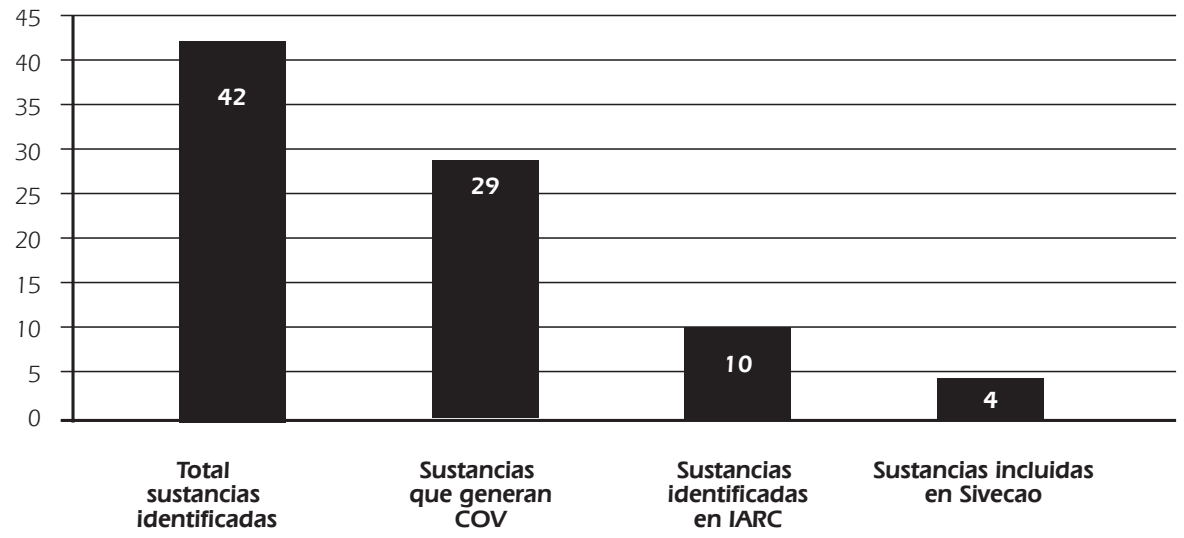

Figura 2. Análisis de las sustancias identificadas en los procesos de pintura de la industria automotriz Fuente: elaboración propia 
como carcinógenas según IARC y 3 en el Sistema de Vigilancia del Cáncer Ocupacional (figura 4).

Por último, en la industria de la construcción (25) fueron identificadas 28 sustancias, de las cuales 14 son COV, 2 se consideran carcinógenas según IARC y 1 por el Sistema de Vigilancia Epidemiológica de Cáncer Ocupacional (figura 5).

La tabla 1 presenta las sustancias identificadas como carcinógenas según IARC y de interés para Colombia según el Sistema de Vigilancia Epidemiológica de Cáncer Ocupacional, en las industrias de estudio.

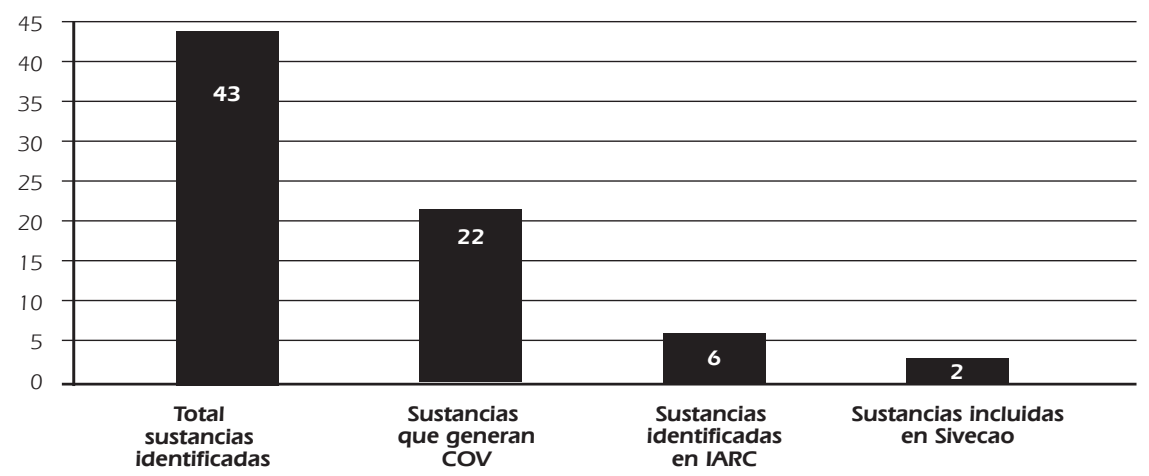

Figura 3. Análisis de las sustancias identificadas en los procesos de pintura de la industria metalmecánica FuENTE: elaboración propia

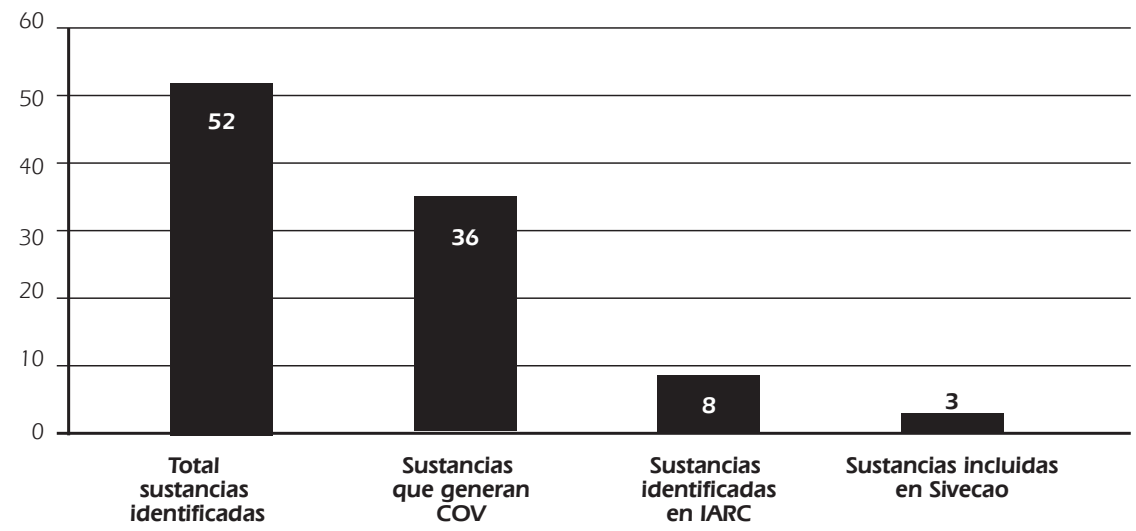

Figura 4. Análisis de las sustancias identificadas en los procesos.

Fuente: elaboración propia 


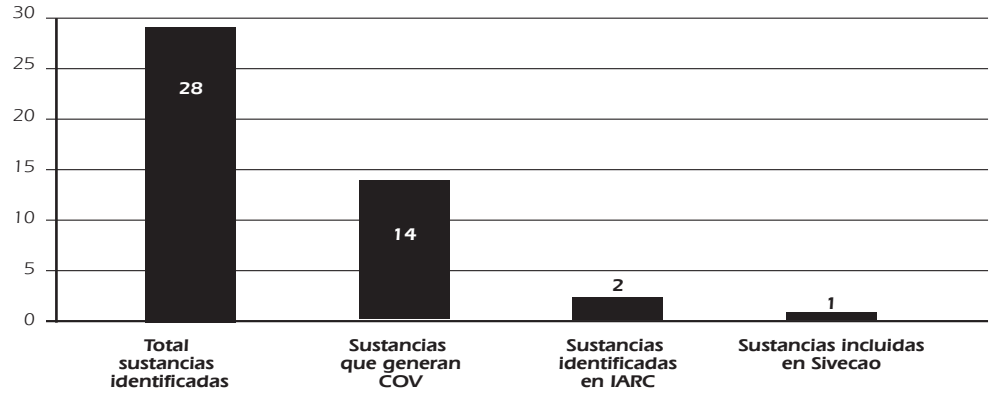

Figura 5. Análisis de las sustancias identificadas en los procesos de pintura de la industria de construcción

FuENTE: elaboración propia

\section{Descripción de la jerarquía de controles}

\section{Sustitución}

La implementación de controles referentes a la sustitución de una sustancia es un proceso que requiere organización, planificación y participación de todas las secciones o departamentos dentro la organización. Existen algunos modelos propuestos por entidades europeas referentes a la sustitución de una sustancia química: la metodología del INRS y el modelo de columnas del IFA (26).

La metodología propuesta por el INRS tiene el objetivo de orientar a las partes interesadas en el proceso de sustitución de agentes químicos peligrosos. Esta metodología parte de que la forma de enfocar la sustitución es, en sí misma, un proyecto; no se trata simplemente de la sustitución de un producto por otro, sino que previamente es preciso un análisis general del problema y de las posibles consecuencias inherentes al realizarla. Esta metodología consta principalmente de los siguientes nueve pasos:

- Identificación del problema.

- Creación del comité de sustitución.

- Estudio y definición de los criterios de selección de sustitutos.

- Opciones de sustitución.

- Ensayos a pequeña escala.

- Evaluación de los nuevos riesgos.

- Comparación y elección del sustituto.

- Implementación.

- Evaluación.

Por su lado, el modelo de columnas de IFA permite comparar de manera fácil y rápida dos sustancias con el fin de decidir cuál de ellas es la más peligrosa. Está dirigido principalmente a la pequeña y mediana empresa (pymes) y a usuarios no especializados. Este modelo consiste en comparar las principales características de peligrosidad de la sustancia que se va a sustituir y de su posible sustituto, y de algunas variables de 
TABLA 1. Sustancias carcinógenas según IARC y el Sistema de Vigilancia del Cáncer Ocupacional (Sivecao)

\begin{tabular}{|c|c|c|}
\hline Industria & IARC & Sivecao \\
\hline \multirow{10}{*}{ Automotriz } & 1,1,2,2-tetracoloretano & Benceno \\
\hline & Benceno & Formaldehído \\
\hline & Cloruro de metileno/diclorometano & Tolueno \\
\hline & Cromato de cinc & Tricloroetileno \\
\hline & Etilbenceno & \\
\hline & Formaldehído & \\
\hline & Isopropilbenceno & \\
\hline & Percloroetileno & \\
\hline & Tricloroetileno & \\
\hline & Vinilbenceno & \\
\hline \multirow{6}{*}{ Metalmecánica } & $\begin{array}{l}4 \text { metil-2-pentanona/isobutil metil cetona/ } \\
\text { metil isobutil cetona }\end{array}$ & Tolueno \\
\hline & Cromato de cinc & Tricloroetileno \\
\hline & Dióxido de titanio & \\
\hline & Gasolina blanca-bencina & \\
\hline & Percloroetileno & \\
\hline & Tricloroetileno & \\
\hline \multirow{8}{*}{$\begin{array}{l}\text { Transformación de } \\
\text { madera }\end{array}$} & 2,4 disocianato de tolueno & Cuarzo en polvo \\
\hline & 2,6 disocianato de tolueno & Formaldehído \\
\hline & $\begin{array}{l}4 \text { metil-2-pentanona/isobutil metil cetona/ } \\
\text { metil isobutil cetona }\end{array}$ & Tolueno \\
\hline & Cuarzo en polvo & \\
\hline & Dióxido de titanio & \\
\hline & Etilbenceno & \\
\hline & Formaldehído & \\
\hline & Vinilbenceno & \\
\hline \multirow{2}{*}{ Construcción } & Alquitrán & Tolueno \\
\hline & Dióxido de titanio & \\
\hline
\end{tabular}

Fuente: IARC-Instituto Nacional de Cancerología. 
proceso, mediante la clasificación de ambos agentes en las correspondientes casillas de una tabla con los siguientes ítems:

- Toxicidad aguda y crónica.

- Riesgo de incendio y explosión.

- Exposición potencial a los peligros.

- Riesgos asociados al proceso.

- Riesgos para el medio ambiente.

La información necesaria para usar este modelo se obtiene de la ficha de datos de seguridad y del conocimiento de las condiciones de uso de la sustancia o compuesto. Adicionalmente, existen nuevas formulaciones que, por su composición o propiedades, son capaces de proporcionar resultados equiparables a las pinturas a base solvente. Algunos ejemplos de estos productos alternativos son: las pinturas al agua (27), las pinturas en polvo (28), las pinturas de alto contenido en sólidos (29) o las pinturas de curación por radiación (30).

El desarrollo de tecnologías en la formulación de productos químicos en base agua y la investigación realizada para desarrollar nuevos tipos de pintura que se ha presentado en las últimas décadas contribuyen notablemente a la sustitución de los COV como principales componentes de los productos químicos empleados en los procesos de pintura y mejoran las condiciones de salud y seguridad de los trabajadores.

\section{Controles de ingeniería}

En cuanto los controles de ingeniería, se utilizan principalmente los métodos de extracción localizada y ventilación general. Para una correcta elección, es necesario conocer las características particulares de la sustancia química, los índices de toxicidad, la concentración de la sustancia en la atmósfera de trabajo, las condiciones particulares en las que se procesa o se manipula, los valores límite permisibles de exposición, así como las principales vías de entrada de la sustancia química al organismo del trabajador. Todos los sistemas y componentes deberán ser diseñados con el único fin de que los contaminantes se mantengan por debajo de los valores limites permisibles.

\section{Extracción localizada}

Este tipo de control (31) es el ideal y efectivo cuando se trabajan sustancias altamente tóxicas, carcinogénicas y peligrosas, cuyo valor límite permisible está por debajo de una parte por millón (ppm). Las sustancias analizadas en los procesos objeto de este estudio que cumplen con esta clasificación se presentan en la tabla 2 .

El sistema de extracción localizada debe contemplar el diseño de elementos como campana, ductos, ventilador y equipos de limpieza, los cuales 
se deben adaptar al proceso. Otros sistemas de captación en los procesos de pintura se centran en encerramientos, cabinas y campanas externas.

Los encerramientos están equipados con pequeñas aberturas de tipo natural en la pared, que permiten que el material se desplace hacia adentro o afuera y proporcione ventilación. El área de estas aberturas debe ser pequeña, comparada con el área total de las paredes del encerramiento (normalmente $5 \%$ o menos).

TABLA 2. Compuestos orgánicos volátiles con valor límite permisible entre 0 y $1 \mathrm{ppm}$

\begin{tabular}{|c|c|c|}
\hline Sustancia & Número CAS & $\begin{array}{c}\text { Valor limite permisible } \\
\text { ACGIH } 2013 \text { (ppm) }\end{array}$ \\
\hline $\begin{array}{l}2,4 \text { diisocianato de to- } \\
\text { lueno }\end{array}$ & $584-84-9$ & $\begin{array}{l}\text { TWA: } 0,005 \\
\text { STEL: } 0,02\end{array}$ \\
\hline $\begin{array}{l}2,6 \text { diisocianato de to- } \\
\text { lueno }\end{array}$ & $91-08-7$ & \begin{tabular}{|l|} 
TWA: 0,005 \\
STEL: 0,02
\end{tabular} \\
\hline Dipropilenglicol metil éter & $34590-94-8$ & $\begin{array}{l}\text { TWA: } 0,1 \\
\text { STEL: ND }\end{array}$ \\
\hline Alquitrán & 65996-93-2 & $\begin{array}{l}\text { TWA: } 0,2 \mathrm{mg} / \mathrm{m}^{3} \\
\text { STEL: ND }\end{array}$ \\
\hline Benceno & $71-43-2$ & $\begin{array}{l}\text { TWA: } 0,5 \\
\text { STEL: } 2,5 \\
\end{array}$ \\
\hline Formaldehído & $50-00-0$ & $\begin{array}{l}\text { TWA: ND } \\
\text { STEL C: } 0,3\end{array}$ \\
\hline
\end{tabular}

Fuente: ACGIH-TLV and BEI's 2013

Las cabinas se caracterizan por que rodean la fuente de emisión, excepto una pared (o porción de esta) que se omite para permitir el acceso a operadores y equipos. El diseño debe prevenir que las partículas incidan en las paredes interiores y la velocidad de captación debe oscilar entre 0,5 y $1 \mathrm{~m} / \mathrm{s}$, para superar la tendencia del aire contaminado que se va a escapar. Algunas de las cabinas utilizadas en los procesos de pintura son:

- Cabinas para humos, vapores y neblinas: el objetivo de este tipo de cabina es contener y expulsar humos, vapores y neblinas. Su funcionamiento se basa en la aspiración del aire desde la parte frontal, el cual es forzado a pasar a través de uno o varios filtros utilizando un motor extractor.

- Campanas tipo cabina para materiales químicos peligrosos: estas se utilizan para materiales químicos de alta toxicidad, es decir, aquellos que tienen un límite permisible entre 0 y 1 ppm, carcinógenos conocidos, sospechosos de carcinógenos o sustancias cuya toxicidad es desconocida.

- Cabinas con cortina de agua: este tipo de cabinas de pintura utiliza un sistema de filtración para eliminar el exceso de pintura en el aire y reducir el consumo de los filtros. Las cabinas de cortina de agua proporcionan una alta eficiencia para remover las partículas de pintura suspendidas en el flujo de aire dentro de la cabina, ya que utiliza agua como medio de filtración. Estas cabinas son utilizadas principalmente para altos caudales de volumen de pintura. 
Las campanas externas se utilizan cuando no es posible encerrar en una cabina el proceso. En este caso, se diseñan las campanas de tal manera que se asegure una velocidad de captura adecuada, que transporte el material contaminante emitido desde su punto más desfavorable hacia ellas.

En el estudio realizado, de las 59 sustancias catalogadas como COV, solo 43 de ellas presentan información referente a los valores límites permisibles por la ACGIH (32), 24 de ellas cuentan con valores por debajo de 100 ppm y 19 se encuentran sobre dicho valor. Así, los controles de ingenieria para este tipo de procesos están enfocados principalmente en la extracción localizada. La tabla 2 presenta los COV identificados, con valores límites permisibles entre 0 y 1 ppm.

\section{Ventilación general o por dilución}

Se recomienda la aplicación de este control (33) cuando en los procesos se manipulen sustancias de baja toxicidad, es decir, cuyo valor límite permisible se encuentre por encima de 100 ppm. Esta ventilación es la que se practica en un área y que renueva todo el volumen de aire con un caudal de aire limpio de procedencia exterior.

El caudal de extracción se debe calcular en función de las renovaciones por hora, las cuales dependen de la naturaleza o características de los lugares de trabajo. Según el ACGIH, para el caso de los talleres de pintura, el número de renovaciones por hora varía entre 30 y 60. Para este cálculo se determina primero el volumen del lugar de trabajo, multiplicando sus dimensiones, así:

$$
\mathrm{V}=\mathrm{L} \times \mathrm{A} \times \mathrm{H}
$$

Donde $V$ es el volumen del lugar de trabajo $\left(\mathrm{m}^{3}\right) ; L$ es el largo del lugar de trabajo (m); A es el ancho del lugar de trabajo (m), y $H$ es la altura del lugar de trabajo $(\mathrm{m})$.

Luego se calcula el caudal multiplicando el número de renovaciones por hora por el volumen del local, mediante la fórmula:

$$
\mathrm{Q}=\mathrm{V} \times \mathrm{N}
$$

Donde $Q$ es el caudal ( $\left.\mathrm{m}^{3} / \mathrm{h}\right) ; V$, el volumen del lugar de trabajo $\left(\mathrm{m}^{3}\right)$, y $N$, el número de renovaciones de aire por hora.

Además de este método de renovación por hora, existe otro recomendado por el ACGIH y que se basa en la cantidad de contaminante generado y en los límites permisibles para calcular el caudal de aire necesario, mediante la siguiente ecuación:

$$
\mathrm{Q}-\mathrm{K} \frac{\mathrm{G} * 10^{3}}{\mathrm{D} * \mathrm{C}}
$$


Donde $Q$ es el caudal de aire necesario $\left(\mathrm{m}^{3} / \mathrm{h}\right)$; $G$ es la tasa de generación del contaminante $(\mathrm{g} / \mathrm{h}) ; D$ es la densidad de vapor del contaminante (g/1); $C$ es la concentración promedio permitida ( $\mathrm{ppm})$, y $K$ es una constante de seguridad que oscila entre 3 y 10, en función de la toxicidad, homogeneidad de la mezcla y volatilidad del contaminante.

La tabla 3 muestra la relación de dilución de los solventes utilizados en los procesos de pintura, recomendados por ACGIH para el diseño de la ventilación.

Tabla 3. Relación de dilución de solventes

\begin{tabular}{|c|c|c|c|c|}
\hline Sustancia & $\begin{array}{c}\text { Densidad } \\
\text { (g/1) }\end{array}$ & $\begin{array}{c}\text { Concen- } \\
\text { tración de } \\
\text { diseño para } \\
\text { la ventilación } \\
\text { (ppm) }\end{array}$ & $\begin{array}{l}\text { Relación de } \\
\text { dilución (m } \\
\text { de aire) por } \\
\mathbf{k g}\end{array}$ & $\begin{array}{l}\text { Relación de } \\
\text { dilución (m } \\
\text { de aire) por } 1\end{array}$ \\
\hline Acetato de butilo & 0,88 & 75 & 2760 & 2520 \\
\hline Acetato de etilo & 0,90 & 75 & 3660 & 3420 \\
\hline Acetato de isopropilo & 0,87 & 75 & 3120 & 2820 \\
\hline Acetato de metilo & 0,96 & 75 & 4320 & 4320 \\
\hline Acetona & 0,79 & 150 & 2760 & 2280 \\
\hline Alcohol butílico & 0,81 & 25 & 12960 & 10920 \\
\hline Alcohol etílico & 0,79 & 250 & 2040 & 1680 \\
\hline Alcohol isopropílico & 0,79 & 150 & 2700 & 2220 \\
\hline Ciclohexanona & 0,95 & 25 & 9780 & 9660 \\
\hline Cloruro de metilo & 0,92 & 200 & 2340 & 2220 \\
\hline Metanol & 0,80 & 100 & 7500 & 6180 \\
\hline Nafta & 0,75 & 200 & 10800 & 840 \\
\hline Tetracloroetano & 1,58 & 5 & 28800 & 47400 \\
\hline Tricloroetileno & 1,46 & 100 & 1800 & 2700 \\
\hline Tolueno & 0,87 & 100 & 2640 & 2340 \\
\hline Varsol & 0,80 & 500 & 360 & 300 \\
\hline Xileno & 0,88 & 75 & 3000 & 2760 \\
\hline
\end{tabular}

Fuente: Manual de Higiene Industrial de MAPFRE

Las sustancias químicas con valores limites permisibles superiores a 100 ppm, utilizadas en los procesos de pintura de las industrias de interés, se muestran en la tabla 4. 
TABLA 4. Sustancias con valores limite permisibles superiores a $100 \mathrm{ppm}$

\begin{tabular}{|c|c|c|}
\hline Sustancia & Número CAS & $\begin{array}{l}\text { Valor limite permisible } \\
\text { ACGIH } 2013 \text { (ppp) }\end{array}$ \\
\hline Alcohol isopropílico & $67-63-0$ & $\begin{array}{l}\text { TWA: } 200 \\
\text { STEL: } 400\end{array}$ \\
\hline Acetona & $67-64-1$ & $\begin{array}{l}\text { TWA: } 500 \\
\text { STEL: } 750\end{array}$ \\
\hline Xileno & $1330-20-7$ & $\begin{array}{l}\text { TWA: } 100 \\
\text { STEL: } 150\end{array}$ \\
\hline Acetato de butilo & $123-86-4$ & $\begin{array}{l}\text { TWA: } 150 \\
\text { STEL: } 200\end{array}$ \\
\hline Acetato de etilo & $141-78-6$ & $\begin{array}{l}\text { TWA: } 400 \\
\text { STEL: No }\end{array}$ \\
\hline Ciclohexanona & $108-94-1$ & $\begin{array}{l}\text { TWA: } 300 \\
\text { STEL: No }\end{array}$ \\
\hline Acetato de metilo & $79-20-9$ & $\begin{array}{l}\text { TWA: } 200 \\
\text { STEL:250 }\end{array}$ \\
\hline Acetato de isobutilo & $110-19-0$ & $\begin{array}{l}\text { TWA: } 150 \\
\text { STEL: No }\end{array}$ \\
\hline Alcohol metílico & $67-56-1$ & $\begin{array}{l}\text { TWA: } 200 \\
\text { STEL: } 250\end{array}$ \\
\hline Butano & $106-97-8$ & $\begin{array}{l}\text { TWA: No } \\
\text { STEL: } 1000\end{array}$ \\
\hline Varsol & $8052-41-3$ & $\begin{array}{l}\text { TWA: } 100 \\
\text { STEL: No }\end{array}$ \\
\hline Metil etil cetona & $78-93-3$ & $\begin{array}{l}\text { TWA: } 200 \\
\text { STEL: } 300\end{array}$ \\
\hline 2-pentanona & $107-87-9$ & $\begin{array}{l}\text { TWA: No } \\
\text { STEL: } 150\end{array}$ \\
\hline Queroseno & $8008-20-6$ & $\begin{array}{l}\text { TWA: } 200 \mathrm{mg} / \mathrm{m}^{3} \\
\text { STEL: No }\end{array}$ \\
\hline Acetato de isopropilo & $108-21-4$ & $\begin{array}{l}\text { TWA: } 100 \\
\text { STEL: } 200\end{array}$ \\
\hline
\end{tabular}

FuENTE: ACGIH-TLV and BEI's 2013.

Para medir la exposición a los contaminantes se deben utilizar los métodos recomendados y publicados en instituciones reconocidas que dispongan de programas de normalización y validación como el NIOSH. Uno de los métodos más efectivos y ampliamente empleado para la caracterización de los ambientes de trabajo que contienen mezclas de COV es el método NIOSH 2549. El procedimiento ha sido capaz de identificar una amplia gama de compuestos orgánicos. La tabla 5 muestra algunos de los compuestos orgánicos más comunes utilizados en los procesos de pintura de las industrias de interés y que son caracterizados por el método. 
TABLA 5. Compuestos orgánicos volátiles identificados por método NIOSH 2549

\begin{tabular}{|l|l|l|l|}
\hline \multicolumn{1}{|c|}{ Sustancia } & \multicolumn{1}{|c|}{ CAS } & \multicolumn{1}{|c|}{ Fórmula } & \multicolumn{1}{c|}{$\begin{array}{c}\text { Peso molecular } \\
\text { (g/mol) }\end{array}$} \\
\hline Benceno & $71-43-2$ & C6H6 & 78,11 \\
\hline Xileno & $1330-20-7$ & C8H10 & 106,70 \\
\hline Tolueno & $108-88-3$ & C7H8 & 92,14 \\
\hline Pentano & $109-66-0$ & C5H12 & 72,15 \\
\hline Hexano & $110-54-3$ & C6H14 & 86,18 \\
\hline Acetona & $67-64-1$ & C3H6O & 58,08 \\
\hline 2-butanona & $78-93-3$ & C4H8O & 72,11 \\
\hline Metil isobutil cetona & $108-10-1$ & C6H12O & 100,16 \\
\hline Metanol & $67-56-1$ & CH3OH & 32,04 \\
\hline Etanol & $64-17-5$ & C2H5OH & 46,07 \\
\hline Isopropanol & $67-63-0$ & C3H7OH & 60,09 \\
\hline Butanol & $71-36-3$ & C4H9OH & 74,12 \\
\hline 2-butoxietanol & $111-76-2$ & C6H14O2 & 118,17 \\
\hline Dietilenglicol etil éter & $111-90-0$ & C6H14O3 & 134,17 \\
\hline Diclorometano & $75-09-2$ & CH2C12 & 84,94 \\
\hline $1,1,1-$ tricloroetano & $71-55-6$ & CC13CH3 & 133,42 \\
\hline Limoneno & $5989-27-5$ & C10H16 & 136,23 \\
\hline Benzaldehído & $100-57-7$ & C7H12O & 106,12 \\
\hline Acetato de etilo & $141-78-6$ & C4H8O2 & 88,10 \\
\hline Acetato de butilo & $123-86-4$ & C7H14O2 & 116,16 \\
\hline
\end{tabular}

Fuente: ACGIH-TLV's and BEI’s 2013

\section{Controles administrativos}

Los controles administrativos recomendados en el estudio se basaron principalmente en los siguientes aspectos:

- Evaluación del ambiente de trabajo (34).

- Vigilancia médica (35).

- Vigilancia ambiental.

- Vigilancia biológica.

- $\quad$ Sistemas de vigilancia epidemiológica (36-38).

- Actividades de promoción y prevención.

- Homologación de los elementos de protección personal.

La evaluación en el ambiente de trabajo se realiza por medio de la implementación, el mantenimiento y la mejora de programas enfocados en prevenir, observar, medir, evaluar y controlar las exposiciones a peligros potenciales para la salud de trabajador. Dependiendo de las características particulares de cada organización y de la problemática específica que se 
plantee, es posible usar, principalmente, tres métodos de vigilancia: medica, ambiental y biológica.

La vigilancia médica busca detectar la presencia o confirmar la ausencia de efectos nocivos para la salud del trabajador (39) como consecuencia de su exposición laboral a sustancias químicas. Esta vigilancia se realiza mediante la aplicación de exámenes médicos y la realización de pruebas paraclínicas y biológicas. Por su parte, la vigilancia ambiental $(40,41)$ documenta la exposición potencial a las sustancias químicas de un grupo de trabajadores, midiendo la concentración de los contaminantes en el ambiente. Por último, la vigilancia biológica evidencia la absorción de las sustancias químicas por el organismo y permite correlacionar estos resultados con los obtenidos durante la medición ambiental, para así obtener la concentración de los contaminantes o sus metabolitos en sangre, orina o aire exhalado por los trabajadores. Sin embargo, tiene sus limitaciones y deberá realizarse únicamente si los objetivos propuestos con la medición ambiental no son alcanzados, ya que implica la realización de procedimientos que requieren la toma de muestras directamente en el trabajador, algunas de ellas de tipo invasivo, como las muestras de sangre.

La decisión de implementar un programa de vigilancia epidemiológica se fundamenta en el análisis integral de la información recopilada mediante las actividades de vigilancia ambiental, médica y biológica, las cuales son la base para implementar los controles administrativos de una manera eficaz.

Las actividades de capacitación, entrenamiento y formación potencian las capacidades individuales de los trabajadores e incrementan la confianza en el momento de realizar las actividades diarias dentro de una organización, y de esta forma contribuyen a la promoción de espacios de trabajo seguros y saludables, fortaleciendo la cultura de autocuidado y toma de conciencia.

La homologación de los elementos de protección personal para manipular sustancias químicas dependerá del tipo de contaminante utilizado, de factores particulares del trabajo, de condiciones propias del trabajador

y de la tarea. En el caso particular de la exposición a COV debe basarse principalmente en una adecuada protección respiratoria y dérmica (42). No obstante, es indispensable el entrenamiento previo sobre el uso y mantenimiento de dichos elementos (43). La tabla 6 muestra los elementos de protección recomendados para el uso de sustancias carcinógenas.

Es importante precisar que cualquier tipo de control que se implemente deberá estar acompañado de actividades de tipo administrativo que complementen las intervenciones realizadas en los ámbitos de control jerárquicamente superiores y que no deberán ser, en ningún caso, las únicas medidas.

\section{Conclusiones}

La medida prioritaria frente a los procesos de pintura en el sector industrial descritos es la sustitución de los COV, los cuales en algunos casos son los principales componentes de la mayoria de los productos 
TABLA 6. Elementos de protección personal recomendados para la manipulación de sustancias carcinógenas

\begin{tabular}{|c|c|c|c|c|}
\hline Sustancia & Protección respiratoria & $\begin{array}{c}\text { Protección } \\
\text { manos }\end{array}$ & $\begin{array}{l}\text { Protección } \\
\text { ocular }\end{array}$ & $\begin{array}{l}\text { Protección piel } \\
\text { y cuerpo }\end{array}$ \\
\hline Alquitrán & $\begin{array}{l}\text { Respirador con filtro } \\
\text { VO si la concentración } \\
\text { es inferior al límite de } \\
\text { tolerancia y si no existe } \\
\text { debilidad de oxígeno. } \\
\text { Respirador con filtro VO } \\
\text { y suplemento de aire si la } \\
\text { concentración es superior } \\
\text { al límite de tolerancia } \\
\text { o existe debilidad de } \\
\text { oxígeno. }\end{array}$ & $\begin{array}{l}\text { Guantes de } \\
\text { nitrilo. } \\
\text { Cremas de } \\
\text { protección. }\end{array}$ & $\begin{array}{l}\text { Utilizar equipa- } \\
\text { miento ocular } \\
\text { cerrado para } \\
\text { proteger de las } \\
\text { salpicaduras de } \\
\text { líquidos. }\end{array}$ & $\begin{array}{l}\text { Vestimenta anti- } \\
\text { estática hecha } \\
\text { con fibras na- } \\
\text { turales o fibras } \\
\text { sintéticas } \\
\text { resistentes a } \\
\text { altas tempera- } \\
\text { turas }\end{array}$ \\
\hline Benceno & $\begin{array}{l}\text { En caso de formarse } \\
\text { vapores/aerosoles, usar } \\
\text { equipo respiratorio con } \\
\text { filtro A. } \\
\text { Equipo autónomo para } \\
\text { muestreo. }\end{array}$ & $\begin{array}{l}\text { Guantes de } \\
\text { nitrilo. }\end{array}$ & $\begin{array}{l}\text { Gafas de segu- } \\
\text { ridad. } \\
\text { Mascarilla del } \\
\text { equipo autóno- } \\
\text { mo. }\end{array}$ & $\begin{array}{l}\text { Bata resistente } \\
\text { a químicos. }\end{array}$ \\
\hline Formaldehído & $\begin{array}{l}\text { Equipo de respiración } \\
\text { autónomo (SCBA) con } \\
\text { máscara completa o con } \\
\text { sistema de protección } \\
\text { positiva. }\end{array}$ & $\begin{array}{l}\text { Guantes de } \\
\text { caucho o } \\
\text { neopreno. }\end{array}$ & $\begin{array}{l}\text { Evitar el uso de } \\
\text { lentes } \\
\text { de contacto; las } \\
\text { lentes blandas } \\
\text { pueden absorber } \\
\text { sustancias irri- } \\
\text { tantes y todas } \\
\text { las lentes las } \\
\text { concentran. }\end{array}$ & $\begin{array}{l}\text { Usar delantales } \\
\text { y ropa protecto- } \\
\text { ra de materiales } \\
\text { como goma } \\
\text { butilo } \\
\text { y nitrilo, polie- } \\
\text { tileno y flúor } \\
\text { carbonato, para } \\
\text { evitar el contac- } \\
\text { to con la piel. }\end{array}$ \\
\hline Percloroetileno & $\begin{array}{l}\text { Máscara con filtro para } \\
\text { vapores orgánicos, que } \\
\text { funcionen a presión por } \\
\text { demanda o con otro sis- } \\
\text { tema de presión positiva, } \\
\text { combinado con equipo } \\
\text { autocontenido. } \\
\text { Cartucho tipo A. }\end{array}$ & $\begin{array}{l}\text { Guantes } \\
\text { largos de } \\
\text { seguridad } \\
\text { de goma } \\
\text { de nitrilo o } \\
\text { alcohol po- } \\
\text { livinílico. }\end{array}$ & $\begin{array}{l}\text { Gafas panorá- } \\
\text { micas contra } \\
\text { salpicaduras o } \\
\text { proyecciones. }\end{array}$ & $\begin{array}{l}\text { Utilizar ropa de } \\
\text { trabajo ade- } \\
\text { cuada que evite } \\
\text { el contacto del } \\
\text { producto. }\end{array}$ \\
\hline Tricloroetileno & $\begin{array}{l}\text { En caso de formarse } \\
\text { vapores/aerosoles, usar } \\
\text { equipo respiratorio con } \\
\text { filtro A, filtro P3. }\end{array}$ & $\begin{array}{l}\text { Usar } \\
\text { guantes de } \\
\text { neopreno o } \\
\text { nitrilo. }\end{array}$ & & $\begin{array}{l}\text { Ropa protectora } \\
\text { impermeable, } \\
\text { que incluya } \\
\text { botas, guantes, } \\
\text { bata de } \\
\text { laboratorio, } \\
\text { delantal. El } \\
\text { material reco- } \\
\text { mendado es el } \\
\text { neopreno. }\end{array}$ \\
\hline
\end{tabular}

Fuente: elaboración propia basándose en hojas de seguridad de las sustancias. 
utilizados. Los controles encaminados a la sustitución de los COV por otras sustancias menos nocivas para la salud de los trabajadores son las que se han desarrollado mayormente en los últimos años. No obstante, la sustitución conlleva varios problemas que hacen que su aplicación presente dificultades, debido a que es difícil encontrar agentes químicos que sean técnica y económicamente viables como sustitutos, además pueden presentar un cierto grado de peligrosidad que siempre se debe considerar y gestionar.

Debido a la gran cantidad de sustancias químicas que se utilizan en los procesos de pintura en las industrias de interés, solo se han establecido los valores límite de exposición y sus métodos de medición para algunas de ellas, lo que restringe la toma de decisiones para implementar los controles necesarios, con el fin de preservar la salud de los trabajadores. Sin embargo, en las situaciones anteriores en las cuales se desconozcan los valores limites permisibles o cuando no se cuenten con los recursos para realizar mediciones ambientales de la concentración de los agentes químicos, el método propuesto es la evaluación simplificada del riesgo del INRS, que es una excelente alternativa a la hora de evaluar el riesgo.

Los controles de ingeniería conllevan incorporar tecnologías usadas normalmente para limitar la diseminación de un peligro en el ambiente laboral y controlar los riesgos para el trabajador; de ahí surge de la necesidad de complementar las acciones correctivas o preventivas tendientes a eliminar o reducir la posibilidad de deterioro en la salud de los trabajadores. Sin embargo, su uso se limita a procesos o sitios específicos, principalmente por razones técnicas y económicas; pero dado que el grado de riesgo es dependiente de la concentración del contaminante y del tiempo de exposición, es imperativo que para reducir el riesgo se tenga que actuar sobre alguno de estos dos parámetros.

La disminución del riesgo hasta valores aceptables se centra en la implementación de controles de sustitución y de ingeniería. No obstante, es necesario aplicar controles de tipo administrativo, dado el diseño de estrategias de muestreo de los contaminantes que permitirian a las organizaciones conocer y comparar las concentraciones con los valores límites permisibles. Igualmente, es necesario diseñar estrategias de prevención de enfermedades laborales, como son los diseños de sistemas de vigilancia epidemiológica y de seguimiento a los biomarcadores.

\section{Recomendación}

Para futuras investigaciones sobre el tema se recomienda realizar un análisis estadístico previo, a fin de determinar cuáles son los productos más usados en el país dentro de las actividades de pintura involucradas en las industrias objeto de este estudio. Ello con el propósito de llevar a cabo un análisis más profundo que identifique con un mayor grado de detalle las sustancias químicas que componen los productos utilizados, su grado de peligrosidad y el impacto que generan en los trabajadores que manipulan estos productos en sus actividades cotidianas. 


\section{Conflicto de interés}

Ninguno.

\section{Referencias}

1. Organización Internacional del Trabajo (OIT). Convenio 170 sobre los productos químicos [internet]. Ginebra; 1990 [citado 30 noviembre 2012]. Disponible en: http://www.ilo.org/ilolex/cgi-lex/convds. $\mathrm{pl}$ ? 170

2. Organización Internacional del Trabajo (OIT). Recomendación 177 sobre la seguridad en la utilización de los productos químicos en el trabajo [Internet]. Ginebra; 1990 [citado 9 abril 2013]. Disponible en: http://www.ilo.org/ilolex/cgi-lex/convds.pl?R177

3. Manku GS. Principios de la química inorgánica. México: McGrawHill; 1983.

4. Chang R. Química. México: s. e.; 1992.

5. Giudice C, Pereyra A. Tecnología de pinturas y recubrimientos componentes. formulación, manufactura y control de calidad. La Plata: Universidad Tecnológica Nacional; 2009.

6. Ríos K. Evaluación simplificada del riesgo químico, a nivel de afectación en salud, seguridad e impacto ambiental en laboratorios de una institución educativa privada en Bogotá D. C. en el periodo comprendido entre noviembre de 2010 hasta octubre de 2011. Bogotá: Pontificia Universidad Javeriana; 2011.

7. Henao F. Riesgos químicos. Bogotá: Ecoe; 2007.

8. Guevara H. Exposición ocupacional a solventes orgánicos en una fábrica de pinturas en Venezuela [Internet]. 1997 [citado 18 marzo 2013]. Disponible en: http://www.bvsde.paho.org/bvsast/e/fulltext/ exposi/exposi.pdf

9. Instituto Nacional de Salud. Sistema Nacional de Vigilancia en Salud Pública [internet]. Bogotá [citado 18 noviembre 2012]. Disponible en: http://www.ins.gov.co/lineas-de-accion/Subdireccion-Vigilancia/sivigila/Estadsticas \%20SIVIGILA/Forms/public.aspx

10. Jiménez F. Intoxicación crónica ocupacional por solventes orgánicos: reporte de un caso clínico. Rosario: Facultad de Medicina de la Universidad del Rosario; 2007.

11. American Industrial Hygiene Association. Norma técnica ANSI Z10 Occupational Health and Safety Managment Systems. 2005.

12. Schweigger E. Manual de pintura y recubrimientos plástico. Madrid: Díaz de Santos; 2007.

13. VOC regulations for coatings: a review and a look forward. J Prot Coat Linings. 2008;1:65-6.

14. Mapfre. Introducción al proceso de pintado de vehículos [internet]. Madrid [citado 30 mayo 2013]. Disponible en: http://www.mapfre. com/ccm/.../CFPreparacionSuperficiesEXTRACTO.pdf

15. National Institute for Occupational Safety and Health (NIOSH). Workplace hazards on female reproductive health [internet]. Atlanta 
[citado 23 octubre 2012]. Disponible en: http://www.cdc.gov/niosh/ docs/99-104/pdfs/99-104.pdf

16. International Agency for Research on Cancer (IARC). [internet]. Lyon [citado 5 agosto 2013]. Disponible en: http://monographs.iarc.fr/ ENG/Classification/

17. Omar M, Viti V, Saito K, Liu J. Self-adjusting robotic painting system. The Industrial Robot. 2006;33(1):50-5.

18. Alcaldía Mayor de Bogotá. Oportunidades de producción más limpia en la industria metalmecánica: guía para empresarios. Bogotá; 2006.

19. Sika. Preparación de superficies metálicas [internet]. [Citado 19 mayo 2013]. Disponible en: http://www.eac.com.co/.../PREPARACION \%20DE \%20SUPERFICIES \%20METALIC

20. Instituto Nacional de Cancerología. Sistema de vigilancia epidemiológica del cáncer ocupacional [internet]. Bogotá [citado 18 noviembre 2012]. Disponible en: http://comisionescanceryocupacion.blogspot. com/2009/12/documento-sivecao-actualizado.html

21. Jensen LK, Larsen A, Molhave L, Hansen MK, Knudsen B. Health evaluation of volatile organic compound (VOC) emissions for wood and wood-based materials. Arch Environ Health. 2001 Sep;56(5):419-32.

22. Instituto Tecnológico del Mueble, Madera, Embalaje y Afines (AIDIMA). Guía de mejores técnicas disponibles para el sector de fabricación de la madera. Valencia; 2009.

23. Pascual Cortés JM. Tintado, glaseado, patinado y difuminado: acabado de carpintería y mueble. s. 1.: IC; 2011.

24. Ruiz G. Riesgo de exposición a compuestos químicos en trabajadores de transformación de la madera [internet]. 2011 [citado 21 mayo 2013]. Disponible en: http://promocionsalud.ucaldas.edu.co/downloads/Revista17(1)_7.pdf

25. Stringent new VOC regulations on architectural and industrial coatings. Pigment \& Resin Technology. 2005;34(2):103.

26. Oleart Comellas P, Pou Serra R, Rabassó Campi J, Sanz Gallén P. Guía práctica para la sustitución de agentes químicos por otros menos peligrosos en la industria. Valencia: Foment del Treball; 2011.

27. Calvo J. Pinturas y recubrimientos: introducción a su tecnología. Madrid: Días de Santos; 2010.

28. Industrias GM. Pintura electrostática [internet]. México D. F. [citado 5 junio 2013]. Disponible en: http://www.igm.mex.tl/imagesnew2/0/0/0/0/.../Pintura \%20Electrostatica.pdf $\square$

29. Tecnolimpia, Consejo Nacional de Producción Limpia (CPL). Guía de mejores técnicas disponibles para el proceso de pinturas automotrices [internet]. Santiago de Chile [citado 20 junio 2013]. Disponible en: http://www.tecnololimpia.cl

30. Secretaría de Medio Ambiente. Alternativas, procesos y/o equipos de reducción de compuestos orgánicos volátiles. Ciudad de México; 2012.

31. Fundación Mapfre. Manual de higiene industrial. s. 1.; 1991. 
32. American Conference of Industrial Hygienists (ACGIH). TLV`s and BEI’s 2013. 7th ed. Cincinnati; 2013.

33. Puerta J, Quinchia R. Ventilación Industrial y equipos de limpieza de aire. 3a ed. Bogotá: Multigráficas; 2003.

34. Organización Internacional del Trabajo. Enciclopedia de salud y seguridad en el trabajo [internet]. Ginebra [citado 15 octubre 2013]. Disponible en: http://www.insht.es/portal/site/Insht/menuitem. 1f1a3bc79ab34c578c2e8884060961ca/?vgnextoid=a981ceffc39a51 10VgnVCM100000dc0ca8c0RCRD\&vgnextchannel=9f164a7f8a6511 10VgnVCM100000dc0ca8c0RCRD

35. Churchill JE, Ashley DL, Kaye WE. Recent chemical exposures and blood volatile organic compound levels in a large population-based sample. Arch Environ Health. 2001 Mar;56(2):157-66.

36. Suk WA, Olden K. Multidisciplinary research: strategies for assessing chemical mixtures to reduce risk of exposure and disease. Hum Ecol Risk Assess. 2005;11(1):141-51.

37. Ministerio de la Protección Social. Guía de atención integral de salud ocupacional basada en la evidencia para cáncer de pulmón relacionada con trabajo (GATISO- CAP). Bogotá; 2007.

38. Ministerio de la Protección Social. Guía de atención integral de salud ocupacional basada en la evidencia para trabajadores expuestos a benceno y sus derivados (GATISO-BTX-EB). Bogotá: 2007.

39. Mizukoshi A, Kumagai K, Yamamoto N, Noguchi M, Yoshiuchi K, Kumano $\mathrm{H}$, et al. A novel methodology to evaluate health impacts caused by VOC exposures using real-time VOC and holter monitors. Int J Environ Res Public Health. 2010;7(12):4127-38.

40. Agencia de Protección Ambiental. Manual de costos de control de contaminación del aire de la EPA. 6a ed. Springfield; 2002.

41. So Y, Ladou J. Diagnóstico y tratamiento en medicina laboral y ambiental. 4a ed. México: Manual Moderno; 2006.

42. 3M. Catálogo de salud ocupacional y seguridad ambiental [internet]. Santiago de Chile. [citado 27 noviembre 2013]. Disponible en http:// www.3mseguridadindustrial.cl/descargas/\#p_respitratoria

43. Occupational Safety \& Health Administration (OSHA). 29 CFR 1910 General Industry; 2012. 